\title{
Unified transform architecture for AVC, AVS, VC-1 and HEVC high-performance codecs
}

\author{
Tiago Dias ${ }^{1,2,3^{*}}$, Nuno Roma ${ }^{1,2}$ and Leonel Sousa ${ }^{1,2}$
}

\begin{abstract}
A unified architecture for fast and efficient computation of the set of two-dimensional (2-D) transforms adopted by the most recent state-of-the-art digital video standards is presented in this paper. Contrasting to other designs with similar functionality, the presented architecture is supported on a scalable, modular and completely configurable processing structure. This flexible structure not only allows to easily reconfigure the architecture to support different transform kernels, but it also permits its resizing to efficiently support transforms of different orders (e.g. order-4, order-8, order-16 and order-32). Consequently, not only is it highly suitable to realize high-performance multi-standard transform cores, but it also offers highly efficient implementations of specialized processing structures addressing only a reduced subset of transforms that are used by a specific video standard. The experimental results that were obtained by prototyping several configurations of this processing structure in a Xilinx Virtex-7 FPGA show the superior performance and hardware efficiency levels provided by the proposed unified architecture for the implementation of transform cores for the Advanced Video Coding (AVC), Audio Video coding Standard (AVS), VC-1 and High Efficiency Video Coding (HEVC) standards. In addition, such results also demonstrate the ability of this processing structure to realize multi-standard transform cores supporting all the standards mentioned above and that are capable of processing the $8 \mathrm{k}$ Ultra High Definition Television (UHDTV) video format $(7,680 \times 4,320$ at $30 \mathrm{fps})$ in real time.
\end{abstract}

Keywords: Video coding; Multi-standard; Transform architecture; Systolic array; FPGA

\section{Introduction}

Multimedia centric devices and applications, especially those based on digital video, have become increasingly popular along the past decade as a result of the latest technological advances. Examples of such systems include video surveillance, IPTV and high definition (HD) TV equipment, as well as the most recent mobile and portable devices, which already offer high-quality video telephony and video conferencing services. Despite their quite distinct characteristics in terms of performance, memory capacity, communication bandwidth and power consumption, nowadays, most of these systems must be capable of processing not only standard definition $(\mathrm{SD})$ contents but also the much more computationally demanding HD contents. Furthermore, they are also required to support several different state-of-the-art video standards, including

*Correspondence: Tiago.Dias@inesc-id.pt

1 INESC-ID, Rua Alves Redol, 9, Lisbon 1000-029, Portugal

${ }^{2}$ IST, Universidade de Lisboa, Avenida Rovisco Pais, 1, Lisbon 1049-001,

Portugal

Full list of author information is available at the end of the article the MPEG-4 Advanced Video Coding (AVC)/H.264 [1], the Audio Video coding Standard (AVS) [2], the VC-1 [3] and the High Efficiency Video Coding (HEVC)/H.265 [4] standards, due to interoperability issues and to guarantee their commercial success. Consequently, video codecs based on highly specialized hardware processing structures have become fundamental building blocks of multimedia systems in several application domains, so as to make them able to efficiently support the encoding and the decoding of a diverse set of contents in real time.

Despite the several existing differences in the adopted coding algorithms, almost all the state-of-the-art video standards implement the classical block-based motioncompensated transform coding scheme [5] and try to maximize the offered compression efficiency for a given video quality. Data compression is mostly achieved in the spatial domain by the transform and quantization stages of the codec, where transforms are used to decorrelate the residual data and compact its energy at the low-frequency domain. In practice, most of the achieved

\section{Springer}

(c) 2014 Dias et al: licensee Springer. This is an Open Access article distributed under the terms of the Creative Commons Attribution License (http://creativecommons.org/licenses/by/4.0), which permits unrestricted use, distribution, and reproduction in any medium, provided the original work is properly credited. 
data compression is provided by the quantization module, which efficiently exploits the reduced sensibility of the human visual system to higher frequency contents, by adaptively coding the obtained transform coefficients.

Nevertheless, transform coding has been an active research topic for several decades, due to the significant impact of this operation in the achieved compression efficiency. This investigation has addressed not only the definition of new transforms to be included in novel video standards but also the design of efficient algorithms and circuits for their computation. As a result, various transforms with distinct sizes and kernels have been presented in the literature, although the majority of such proposals are based on the type II order-8 discrete cosine transform (DCT) [6]. This is owed to the reduced complexity and the highly satisfactory performance of the DCT in terms of energy compaction, as well as to the existence of fast algorithms for its computation [6].

Nevertheless, transforms of different orders (i.e. order2 , order-4, order-16 and order-32) are nowadays also considered by the most recent video standards (e.g. AVC, AVS, VC-1 and HEVC), in order to improve the coding efficiency, both for HD videos and for low-complexity mobility applications. These smaller transforms are more suitable to process the smaller block sizes resulting from the application of enhanced Inter and Intra prediction techniques in the encoding of low-resolution videos, since they allow to improve the energy compaction and reduce the quantization error. Conversely, larger transforms allow to greatly increase the compression rate for HD contents. However, this higher compression efficiency also comes at the expense of increased computational complexity and required data processing performance. This poses several challenges to codec and system designers, especially when real-time operation is demanded. This issue is even more problematic for the newest multimedia devices, which are required to support several different video standards. In fact, to implement the several different video codecs, they must efficiently compute the corresponding set of two-dimensional (2-D) transforms. Consequently, the investigation of high-performance and area-efficient multi-standard transform (MST) architectures is nowadays a very active research area.

In this paper, a unified architecture is proposed for the implementation of high-performance MST cores. The proposed scalable and configurable hardware structure is based on a 2-D systolic array transform core, which was recently proposed for the computation of the transforms adopted in the AVC and HEVC standards [7]. Such transform core presents a modular and flexible interconnection architecture that not only allows it to be reconfigured to support different transform kernels but also permits its rescaling to efficiently support transforms of multiple sizes. In this work, such properties are extensively exploited to propose individual transform cores for the AVC, AVS, VC-1 and HEVC standards. Moreover, a MST core capable of processing the transforms defined in all these standards in a single hardware structure is also presented. To achieve such goal, several new processing elements (PEs) with both dedicated and multistandard computation capabilities are also introduced herein.

The rest of this paper is organized as follows. Section 2 provides a review of the set of transforms adopted in the previously mentioned video standards, while the most relevant related work is introduced in Section 3. Section 4 presents the proposed MST architecture, as well as the set of PEs that are used to compute the AVC, AVS, VC-1 and HEVC transforms. The experimental results considering the implementation of these PEs and of a MST transform core in a Xilinx Virtex-7 FPGA device are presented and discussed in Section 5, by considering other state-of-theart designs with similar functionalities. Finally, Section 6 concludes the presentation.

\section{Transform coding}

The type II DCT [6] has been extensively used in blockbased image and video coding, such as in the JPEG, H.261/3 and MPEG-1/2/4 standards. This is mainly a consequence of its modest complexity and robust approximation to the statistically optimal Karhunen-Loève transform [5], which allows to obtain implementations with good performance, both in terms of computation time and energy compaction.

Several different algorithms for fast and efficient computation of the DCT have been developed [6]. Many of them take advantage of the existing relationships between the DCT and various other fast transforms, while others are based on the sparse factorization of the DCT kernel. Alternative proposals consist of recursive implementations, like the the row-column decomposition strategy, which poses several advantages in terms of complexity, computation time and design effort. Nevertheless, the computation of the simpler one-dimensional (1-D) transforms still presents several challenges, since some entries of these transform kernels consist of irrational numbers: on the one hand, because its computation requires either floating-point calculations or integer approximations using high-precision coefficient values, which both lead to the usage of expensive and rather slow arithmetic circuits, and on the other hand, due to the drift that may be introduced in the coding procedure, i.e. mismatch between the decoded data in the encoder and decoder. This can result from the forward and inverse transforms being implemented in different machines offering distinct floating-point representations, approximations or rounding procedures. This is a critical problem for modern video standards (e.g. AVC, AVS, VC-1 and HEVC), 
since they make an extensive use of prediction techniques to increase their compression efficiency [1-4], thus becoming particularly sensitive to the prediction drift.

To circumvent this problem, state-of-the-art video standards have adopted integer transforms with almost the same symmetry and energy compaction capability than the DCT. Although several different approaches can be used to obtain such transform kernels, dyadic symmetry schemes [8] have been followed in almost all the standards. This approach not only allows to compute the transform in reduced bit-width integer arithmetic (e.g. 16 bits) but also exclusively using integer multiplications and additions. However, since the basis vectors of these integer transforms do not have the same norm, this approach often implies an additional normalization stage in the video coding algorithm. In many video standards, this compensation has been relegated to the quantization stage of the video codec, in order to keep the complexity as low as possible $[1,3,4]$.

In the following subsections, the transform processes that are employed by the most relevant state-of-the-art video standards are briefly reviewed.

\subsection{MPEG-4 AVC/H.264}

AVC [1] considers a two-level hierarchical transform process involving six different transforms, namely, the forward and the inverse $8 \times 8$ and $4 \times 4$ DCTs and the $4 \times 4$ and $2 \times 2$ Hadamard transforms. In the first transform level, the $4 \times 4$ DCT is applied to all the $4 \times 4$ luma and chroma blocks of residual data. The kernels of these forward and inverse transforms, generically represented in (1), contain only three different values each, as enumerated in Table 1. Such characteristic greatly minimizes the complexity of the transform computation procedure, by allowing its

\section{Table 1 Coefficients of the AVC, AVS, VC-1 and HEVC transform kernels}

\begin{tabular}{|c|c|c|c|c|c|c|c|}
\hline \multirow[t]{2}{*}{ Transform } & \multicolumn{7}{|c|}{ Kernel values } \\
\hline & $a$ & $b$ & $c$ & $d$ & $e$ & $f$ & $g$ \\
\hline AVC $2 \times 2$ Hadamard & 1 & - & - & - & - & - & - \\
\hline AVC $4 \times 4$ Hadamard & 1 & - & - & - & - & 1 & 1 \\
\hline AVC $4 \times 4$ forward DCT & 1 & - & - & - & - & 1 & 2 \\
\hline AVC $4 \times 4$ inverse DCT & 1 & - & - & - & - & 1 & $\frac{1}{2}$ \\
\hline AVC $8 \times 8$ inverse DCT & 8 & 12 & 10 & 6 & 3 & 8 & 4 \\
\hline AVS $4 \times 4 \mathrm{DCT}$ & 2 & - & - & - & - & 3 & 1 \\
\hline AVS $8 \times 8 \mathrm{DCT}$ & 8 & 10 & 9 & 6 & 2 & 10 & 4 \\
\hline VC-1 $4 \times 4$ DCT & 64 & - & - & - & - & 83 & 36 \\
\hline VC-1 $8 \times 8$ DCT & 12 & 16 & 15 & 9 & 4 & 16 & 6 \\
\hline HEVC $4 \times 4$ DCT & 64 & - & - & - & - & 83 & 36 \\
\hline HEVC $8 \times 8$ DCT & 64 & 89 & 75 & 50 & 18 & 83 & 36 \\
\hline
\end{tabular}

computation using only 16-bit integer addition and shift operations.

$$
C_{4 \times 4}=\left[\begin{array}{rrrr}
a & a & a & a \\
f & g & -g & -f \\
a & -a & -a & a \\
g & -f & f & -g
\end{array}\right]
$$

The second level of the transform process is based on the Hadamard transform and strictly concerns the processing of the DC coefficients of the higher level transform blocks. Two distinct Hadamard transforms are used to process the coefficients corresponding to the luma and chroma blocks. For the macroblocks (MBs) that are encoded using the $16 \times 16$ Intra prediction mode, a $4 \times 4$ Hadamard transform is used to process the 16 DC coefficients of the luma blocks. Conversely, a $2 \times 2$ Hadamard transform is used to process the chroma DC coefficients in all coding modes. The kernel of this Hadamard transform consists of a subsampled version of the $4 \times 4$ kernel, which considers only the first two entries of rows 0 and 2. Both transforms can be computed by exclusively using integer additions and subtractions, since their kernels contain only the 1 and -1 values (see Table 1 ). Due to the symmetric nature of the two kernels, the inverse $4 \times 4$ and $2 \times 2$ Hadamard transforms use the same kernels of their corresponding forward transforms.

The High profiles defined in the Fidelity Range Extension (FRExt) amendment of the AVC standard also allow the processing of MBs composed of luma blocks with $8 \times 8$ samples [1]. In such cases, the transform process consists only of the first transform level. Furthermore, a single $8 \times 8$ integer transform is used to process the four luma blocks. Just like the Hadamard transforms, the forward and inverse $8 \times 8$ DCTs also share the same kernel. However, the computational complexity of these procedures is greater than that of the $4 \times 4$ kernels, since they imply a larger and more diverse set of kernel values (see (2) and Table 1). In addition, the involved circuit complexity is also higher, due to the greater magnitude of the considered kernel values (see Table 1 ). The resulting dynamic gain of the $8 \times 8$ kernels is therefore relatively higher, which imposes an intermediate rescaling after the row-wise 1-D transform stage, in order to make its computation possible with 16-bit integer arithmetic.

$$
C_{8 \times 8}=\left[\begin{array}{rrrrrrrr}
a & a & a & a & a & a & a & a \\
b & c & d & e & -e & -d & -c & -b \\
f & g & -g & -f & -f & -g & g & f \\
c & -e & -b & -d & d & b & e & -c \\
a & -a & -a & a & a & -a & -a & a \\
d & -b & e & c & -c & -e & b & -d \\
g & -f & d & -g & -g & f & -f & g \\
e & -d & c & -b & b & -c & d & -e
\end{array}\right]
$$




\subsection{VC-1}

One of the main goals of the VC-1 standard [3], which was initially developed by Microsoft Corporation as WMV-9 and later standardized by the Society of Motion Picture and Television Engineers (SMTPE), is to provide efficient codec implementations for online video services. This type of video services usually requires low-complexity codecs that must be capable of producing low bit-rate video streams. To comply with both requirements, VC-1 also implements a hybrid motion-compensated and transform coding scheme. However, the complexity of this coding procedure is much lower than the one adopted in the AVC standard.

In what concerns the transform process, $\mathrm{VC}-1$ also makes use of variable-size transforms to better exploit the spatial correlation of the data and to improve the coding efficiency. However, it only considers a single transform level and two distinct kernels to process all the possible block sizes, i.e. $8 \times 8,8 \times 4,4 \times 8$ and $4 \times 4$ (see (1), (2) and Table 1). Intra MBs are always encoded using the $8 \times 8$ transform. However, Inter MBs can be encoded using any of the four available separable transforms, provided that the optional variable-size transform mode has been enabled in the encoder.

The VC-1 transform process also allows fast algorithm implementations to compute the inverse transforms. These transforms, which do not involve any rescaling operations, can be realized using 16-bit arithmetic and without any multiplications. Conversely, the computation of the forward transforms is slightly more complex, due to requiring an extra normalization stage to compensate the different norms of its basis functions. Similarly to the AVC standard, such scaling factors are also absorbed by the quantization procedure [3].

\subsection{AVS}

AVS [2] was developed in China and adopts a coding scheme analogous to AVC, which is why the two standards offer quite similar coding performances. Nevertheless, contrary to what happens with AVC, the transform process of AVS considers the computation of a single 2-D transform for the processing of all the luma and chroma blocks that compose a $16 \times 16$ pixel MB.

In AVS Part 2 (a.k.a. AVS 1.0), which targets highdefinition digital video broadcasting and high-density storage media, a MB is composed of four $8 \times 8$ luma blocks and of two chroma blocks with $8 \times 8$ samples each. All these blocks are processed by an $8 \times 8$ integer transform that was designed in conjunction with the quantization process by using the pre-scaled integer transform (PIT) technique [9], in order to reduce the rounding errors and minimize the complexity of the decoder implementation. Such 2-D transform consists of a separable and integer-precise transform, which can be computed using 16-bit arithmetic, thanks to the reduced magnitude of the involved kernel values (see Table 1). However, rounding and rescaling operations must be applied after the computation of the row and column transforms, not only to guarantee the accuracy of the 16-bit operation but also to harmonize the transform/quantization and entropy coding procedures.

The alternative Part 7 of AVS, commonly known as AVS-M, was proposed for video communication applications targeting mobile devices. Typically, these devices deal with lower resolution videos and possess limited processing capabilities and memory resources. To better adjust the complexity of the video codec to these characteristics, AVS Part 7 defines the $4 \times 4$ block size as the basic unit for the transform process. Accordingly, it specifies a 2-D separable transform for the processing of all the luma and chroma blocks. Due to the considered kernel values (see Table 1 ), such $4 \times 4$ integer transform also can be computed in 16-bit arithmetic and by using only integer additions and shift operations.

\section{$2.4 \mathrm{HEVC} / \mathrm{H} .265$}

In HEVC [4], the basic units defined for the transform and quantization processes are called transform units (TUs). The TUs consist of square blocks of data that can be recursively subdivided in four equally sized blocks, starting from the $32 \times 32$ samples TU format and going all the way down to a minimum of $4 \times 4$ samples. As a result of this segmentation, several different integer transforms with multiple sizes are specified for the HEVC transform process. Nevertheless, all the transform kernels are better approximations of the DCT than those that were adopted by the AVC standard.

The HEVC transform kernels were defined by approximating scaled DCT basis functions under specific considerations, such as limiting the necessary dynamic range for transform computation or maximizing the precision and closeness to orthogonality, whenever the kernel entries are specified as integer values. Consequently, the basis vectors of these transforms have equal energy and there is no need to compensate for the different norms, as in previous video standards. Furthermore, such property also allows to use the same kernels for the computation of both the forward and inverse transforms. However, due to the increased dynamic range of the involved transform kernels, HEVC explicitly inserts rescaling and 16-bit clipping operations after the row-wise transform stage. This guarantees that all the transforms can be computed by using 16-bit integer arithmetic.

To reduce the complexity of the encoder and to simplify the computation of the transforms, only one order32 transform kernel is specified in HEVC (see [4]). The remaining lower order kernels consist of subsampled versions of this kernel. The entries of such order- $k$ kernels 
(with $k=4,8,16$ ) consist of the first $k$ values of rows $k \times j$ (with $j=0 \ldots \frac{32}{k}-1$ ) of the $32 \times 32$ kernel. All the lower order kernels also present key symmetry properties, to enable fast 'partially factored' implementations using very few mathematical operations. As an example of this feature, the HEVC $8 \times 8$ transform kernel can be obtained from (2), by considering the corresponding coefficients enumerated in Table 1.

\section{Related work}

In the last few years, several different transform cores have been presented in the literature. The majority of these solutions consist of dedicated processing structures targeting efficient VLSI realizations in ASICs. However, some alternative proposals addressing the design of specialized programmable processors [10], applicationspecific instruction set processors (ASIPs) [11] and, more recently, efficient implementations in reconfigurable platforms [12] have also been presented. Independently of the considered platforms and technologies, these designs generally implement fast and optimized algorithms and are based on high-performance processing structures, in order to mitigate the involved complexity constraints and speed up the inherent computations. Such operations can concern the realization either of a single transform or of multiple and distinct transforms when multi-transform and multi-standard functionality are desired.

Direct 2-D processing structures are usually employed when high-performance implementations are desired. Such implementations are characterized by their highly parallel processing capability, which allows them to simultaneously compute several different transform coefficients. However, they are also characterized by imposing higher hardware cost and power consumption requirements. As a consequence, not so many direct 2-D transform architectures have been proposed. The most well known of those direct 2-D structures consists of the butterfly architecture proposed by Chen et al. [13] for the computation of the $8 \times 8 \mathrm{DCT}$, adopted in the JPEG image standard and in the MPEG-1/2/4 and H.261/3 video standards. Other direct 2-D designs targeting the most recent standards have also been devised by using quite similar approaches, like the ones presented in $[14,15]$ for the computation of the $4 \times 4$ transforms defined in AVC.

The technique introduced by Chen et al. has also been frequently considered in the development of architectures for the computation of 1-D transforms. With some transposition logic, these simpler processing structures are usually employed to compute 2-D transforms by using the row-column decomposition approach. The hardware cost of these designs is therefore relatively lower, since the same 1-D transform circuit can be used twice in the computation of a 2-D transform. Moreover, the reduction in the hardware cost also contributes to decrease the power consumption associated with this type of architectures. Nonetheless, such gains are usually modest in most practical cases, owing to the usage of quite large and complex memory-based circuits to realize the transposition operation. In addition, these circuits also introduce some latency and slightly restrict the data processing rates for the computation of the 2-D transforms, which may compromise the implementation of real-time operations. Still, many designs of direct 1-D architectures have been proposed to compute 2-D transforms [16].

In what concerns the set of integer transforms adopted by the most recent video standards, such as AVC, AVS, VC-1 or HEVC, some 1-D structures have also been proposed for the computation of the forward and inverse transforms $[17,18]$. Other alternative cost-effective architectures based on serial algorithms were described in [19], while the implementation of designs based on the residue number system (RNS) [20] and in systolic array structures [7] has also been reported. In addition, Coordinate Rotation Digital Computer (CORDIC) arithmetic was also used in the design of some of these structures, not only to avoid the multiplication operations but also to improve the performance and hardware efficiency of their implementation [21]. Other designs making use of distributed arithmetic also to avoid the computation of multiplications have also been proposed [22].

Contrasting with the previously referred dedicated transform cores, the majority of the hardware structures that have been proposed for the newest video standards consist of a distinct class of processing structures, denominated as multi-transform architectures. In particular, when such more elaborated designs are only capable of computing the set of transforms exclusively employed by a specific video standard, they are subclassified as unified transform cores [23-26]. However, if they are designed to implement the several different transforms used by various standards, they are subclassified as multi-standard transform (MST) cores [27-29]. In order to achieve both goals, a couple of different approaches have been considered to devise this type of architectures.

The simplest designs consist of hybrid architectures [29], which make use of a distinct, independent and specifically optimized hardware structure to compute each of the considered transforms. Then, the proper transform results are selected, according to the type of transform that is required. Since all the transforms are computed in parallel, the involved hardware cost, memory bandwidth and power consumption requirements are very high and often prohibitive for most implementations. As a result, several alternative architectures featuring the sharing of hardware resources have been presented to implement more efficient multi-transform processing structures $[27,30,31]$. Some of these designs were even derived by jointly optimizing the algorithms of the considered set 
of transforms using different techniques (e.g. matrix factorizations [19], matrix decompositions [32] and 1-D delta matrix mapping [33]).

Among all the reviewed transform architectures, the design proposed in [7] presents two distinctive properties (i.e. modularity and scalability) that can be successfully exploited to design high-performance and hardwareefficient dedicated and multi-standard transform cores. As a result, such hardware structure was used as the basis for the work that is herein presented.

\section{Unified architecture for MST cores}

The unified architecture for the computation of 2-D transforms herein presented was designed to fully address the data processing requirements of modern video codecs. Consequently, it can be used not only to realize highperformance and hardware-efficient transform cores that are capable of computing specific 2-D transforms but also to implement MST cores supporting the state-of-the-art video standards. To compute such a vast set of transforms, the proposed architecture adopts, as its base structure, the multi-transform core that was presented in [7]. However, the modularity characteristic of such base structure is herein extensively exploited, in order to further extend its functionality by giving it the capability to support the four current state-of-the-art video standards, i.e. AVC, AVS, VC- 1 and HEVC.

In the following subsections, the processing structure that was used as the basis for this work is briefly reviewed. Then, the architecture of a new set of PEs targeting the AVC, AVS, VC-1 and HEVC standards is introduced. Finally, a resource-shared multi-standard PE structure is presented for the processing of all the involved DCTs in MST cores.

\subsection{Base architecture}

The MST core presented in [7] is already able to compute all the $2 \times 2,4 \times 4$ and $8 \times 8$ transforms defined in both the AVC and HEVC standards, by implementing a row-column decomposition strategy. To achieve such goal, this processing structure makes use of the four functional modules depicted in Figure 1: a 2-D systolic array, a transposition switch, an input buffer and a control unit.

The heart of this architecture consists of the $N \times N$ PE systolic array that is used to compute 1-D transforms. Within this scalable structure, the data is processed by the PEs in a wavefront manner, following a regular data streaming model. Accordingly, the data is fed into the PE rows through the input buffers in the left column of the array. Then, it is processed by each PE (as it is explained in Section 4.2) and subsequently propagated in the horizontal and vertical directions to the neighbour PEs inside the array, advancing one PE level (in both directions) at each clock cycle. Conversely, the control signals for all the PEs enter the array through the top left corner PE and are propagated to the other PEs (also in both directions) synchronously with the data propagation. This processing scheme allows to maximize the data processing rate, because it is possible to start the computation of a different transform value in each row of the array on each clock cycle (provided that the input buffers are not empty).

The row-column data transposition is realized by the transposition switch. Unlike other known transposition units [25-27,34], this circuit does not use additional memory resources. In fact, it mostly consists of a scalable crossbar switching circuit composed of $N$ distinct $(N: 1)$ multiplexers, where $N$ corresponds to the size of the instantiated systolic array. Therefore, this design allows a fast and direct transposition of the data.

The input buffer was also designed as a scalable hardware structure that can be configured to operate using $N$ FIFO buffers of depth $N$. It is used to feed the systolic array with the data to be processed, which may consist of (i) the residues from the Intra and Inter predictions, when forward transforms are considered, or (ii) the transform coefficients, when inverse transforms must be computed. By using this module, the data values are serially and smoothly transferred to the several rows of the array, therefore supporting the desired regular and pipelined dataflow within this systolic structure.

The input buffer also provides a reduction of the inherent delays when accessing the external frame memories where the data is stored, since it is operated in parallel with the remaining architecture modules. In addition, it supports two distinct memory access patterns, so as to further optimize such memory accesses. On the one hand, it can be configured to fetch, in parallel, the $N$ data values comprehending an entire row of the block under processing from the frame memories. This strategy is suitable for custom multimedia systems and allows to maximize the data processing rate of the implemented MST core. On the other hand, it can implement a sequential memory access pattern, in which all the data values of the row of the block under processing are loaded into the FIFOs in $N$ subsequent memory accesses. This alternative approach is more convenient when integrating the MST core as a hardware accelerator in multimedia systems based on general-purpose processors. In such cases, the sequential memory access strategy also allows to better exploit cache access patterns, since the $N$ data values composing the block rows to be processed are typically stored within the same cache line.

Finally, the control unit is responsible for controlling the systolic array, as well as the operation of both the input buffer and the transposition switch. It is also in charge of implementing the necessary synchronization mechanisms between the transform core and the outer video 


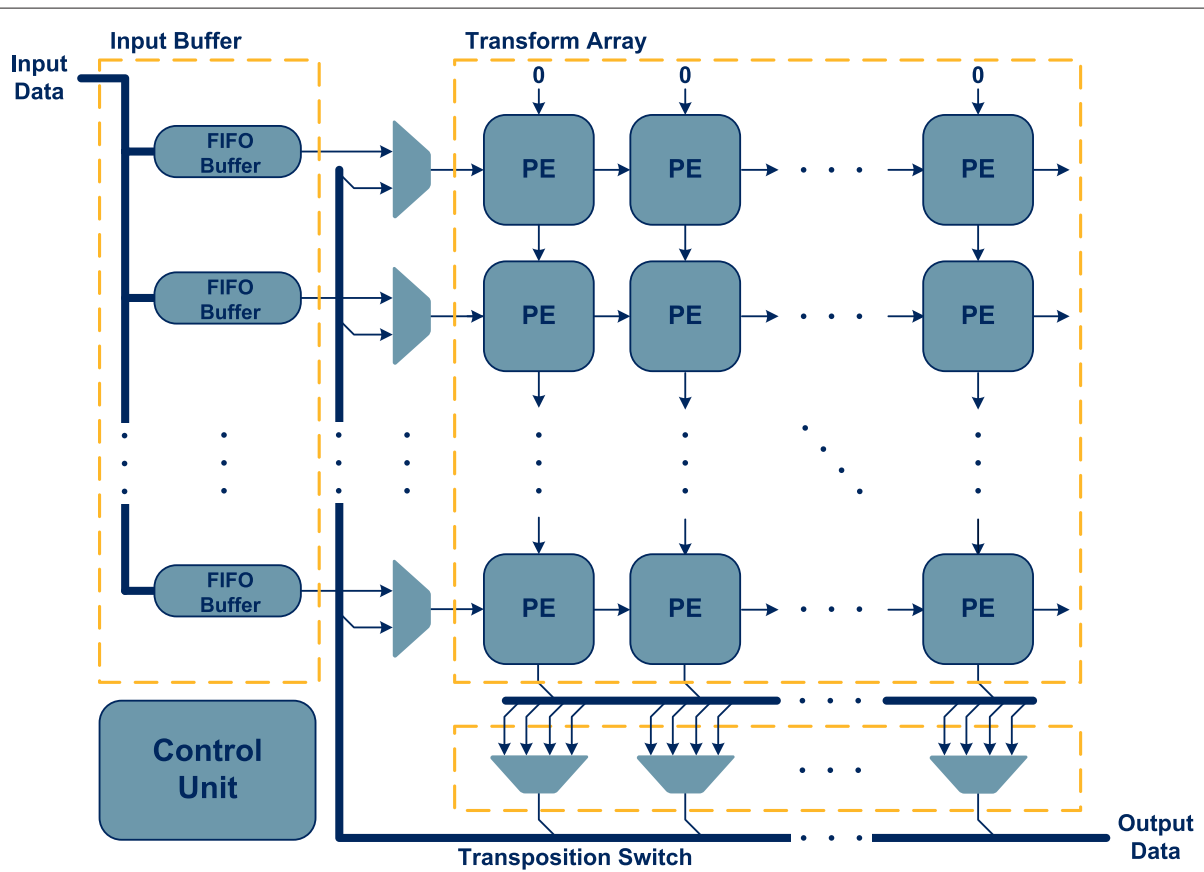

Figure 1 Block diagram of the architecture presented in [7].

coding system that incorporates this dedicated processing structure, as it is explained in [7].

\subsection{PE structure and functionality}

The PEs are the basic units of the proposed unified transform architecture and are responsible for realizing all the operations involved in the computation of the considered 1-D transforms. Within the systolic array, all the PEs share the same architecture and perform the same set of operations, which mostly consist of the multiply-andaccumulate (MAC) operations involved in the transform computation procedure. As it can be seen in Figure 2, which depicts the generic block diagram of a $\mathrm{PE}$, the architecture of these processing structures is composed of two main blocks: a control module and an arithmetic module.

The control module is responsible for guaranteeing the correct flow of all the control signals of the architecture inside the systolic array, giving support to the dataflow model described in Section 4.1. Furthermore, it is also responsible for generating the control signals that command the transform computation procedure inside the $\mathrm{PE}$. One of the key operations that is realized by this module consists in the generation of the multiplier values (i.e. the transform kernel values) to be used in the MAC operations that are performed in the PE. Such values are generated by taking into consideration the horizontal and vertical coordinates of the PE inside the systolic array, as well as the type of transform that must be computed.
The rationale behind the algorithm that was implemented to generate the kernel values for any given DCT results from the observation that only $N-1$ different basis values exist in a $N \times N$ transform kernel [6]. These values consist of the first column of the transform kernel (e.g. see (1) and (2)), which correspond to the subset of kernel values matching the DCT cosine angles in the range $\left[0, \frac{\pi}{2}\right]$. Consequently, they can also be used to generate the remaining $(N-1) \times(N-1)$ values of the transform kernel, provided that the symmetry and periodicity trigonometric properties of the cosine function are properly exploited. This is illustrated in Figure 3 for the case of the generic $4 \times 4$ transform kernel presented in (1), where the value located at position $(2,2)$ of the transform kernel $(-a)$ is obtained by reducing the corresponding angle $(\beta)$ to an elementary angle $\left(\beta^{\prime}\right)$ located in the first quadrant of the trigonometric circle.

To implement the devised algorithm, the control module of the PEs makes use of a quite simple combinational circuit (identified as Multiplier Decoder in Figure 2) and of a small ROM. Together, they generate all the multiplier values corresponding to all the kernel values of the considered transforms. The operation of such circuit for a $N \times N$ transform kernel is the following.

First, the vertical $(r)$ and the horizontal $(c)$ coordinates of the PE inside the systolic array are used to determine the amount $(f)$ of $\frac{\pi}{2 N}$ angular segments of the cosine angle corresponding to the considered kernel value (e.g. $f=(2 c+1) \times r$ for the computation of the row-wise 


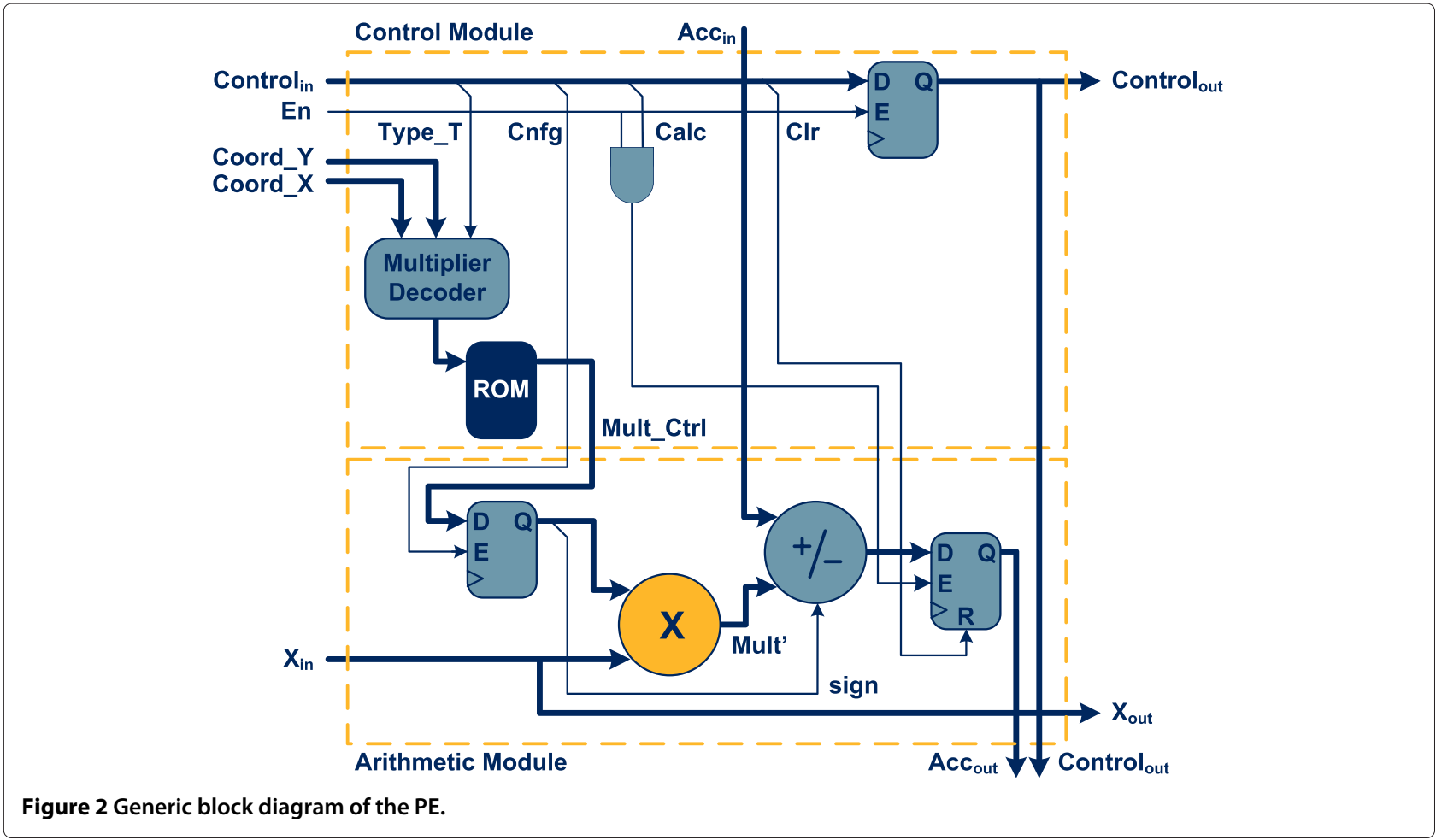

transforms), as specified in the generic formulation of the 2-D DCT shown in (3).

$$
\begin{aligned}
X_{k, l}= & \sqrt{\frac{2}{N}} \epsilon_{k} \epsilon_{l} \sum_{i=0}^{N-1} \sum_{j=0}^{N-1} x_{i, j} \times \cos \left[(2 i+1) \frac{k \pi}{2 N}\right] \\
& \times \cos \left[(2 j+1) \frac{l \pi}{2 N}\right], k, l=0, \ldots, N-1
\end{aligned}
$$

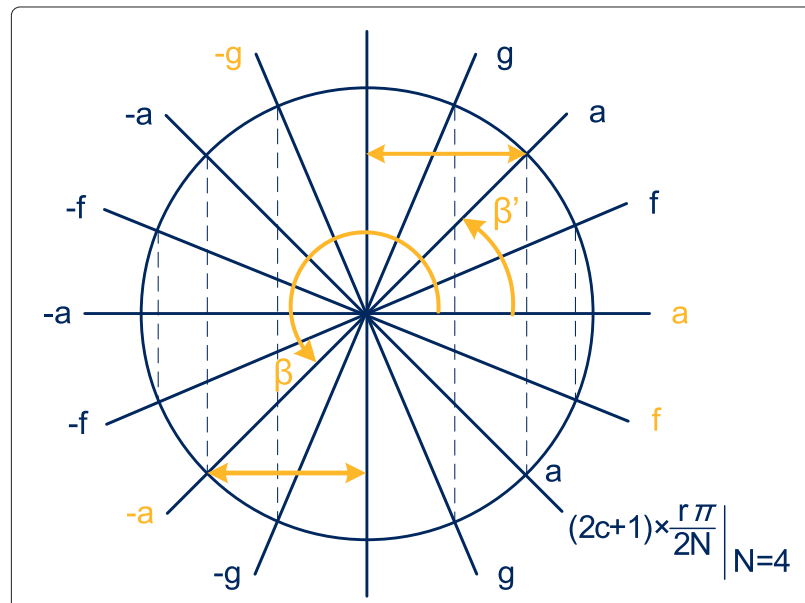

Figure 3 Definition of a transform kernel value. Definition of the value $(-a)$ located at position $(2,2)$ of the generic $4 \times 4$ transform kernel shown in (1), according to the proposed algorithm.

$$
\epsilon_{t}=\left\{\begin{array}{l}
\frac{1}{\sqrt{2}}, \text { if } t=0 \\
1, \text { otherwise }
\end{array}\right.
$$

Although such computations should be performed with integer arithmetic by using $2 \times\left\lceil\log _{2} N\right\rceil+1$ bits, only the $\left\lceil\log _{2} N\right\rceil+2$ least significant bits of $f$ are of practical interest, because they identify the corresponding angular value constrained to the range $[0,2 \pi[$. The remaining bits only specify the amount of times the $2 \pi$ domain was exceeded and therefore can be ignored. To exemplify this procedure, Figure 3 illustrates the definition of all the angles corresponding to the entries of the generic order- 4 transform kernel $(N=4)$ presented in (1). In particular, the angles corresponding to all the values in the second column of this matrix $(c=2 ; r=0 \ldots 3)$ are also highlighted in yellow colour $\left((r, c) \rightarrow \beta=f \times \frac{\pi}{2 \times 4}\right)$.

The two most significant bits of the obtained constrained result $\left(f^{\prime}=f_{<\log _{2} N+1: 0>}\right)$ are then evaluated, in order to determine if the corresponding angle is in one of the following ranges $\left[\frac{\pi}{2}, \pi\right],\left[\pi, \frac{3 \pi}{2}\right]$ or $\left[\frac{3 \pi}{2}, 2 \pi\right]$. In such cases, the constrained value $f^{\prime}$ is computed, which corresponds to a reduction of the original angle into the range $\left[0, \frac{\pi}{2}\right]$. This final result is used to address the ROM, in order to retrieve the required multiplier control word. Such data, which corresponds to the absolute value of the considered transform kernel entry, is then used to control the operation of the specialized multiplication circuit that is embedded in the PE arithmetic module, as it is described below and is represented in Figure 2. 
Finally, the sign information of the kernel value is computed by considering the two most significant bits of $f^{\prime}$. If such data corresponds to an angle in the range $\left[\frac{\pi}{2}, \frac{3 \pi}{2}\right]$

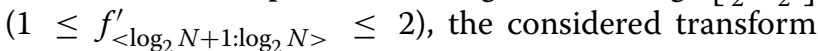
kernel entry represents a negative number. Otherwise, it represents a positive number.

As it can be seen in Figure 2, the obtained multiplier control word and the corresponding sign information bit are not directly applied to the multiplication circuit embedded in the PE. Instead, such data is stored in an internal data-standing register of the PE, so that it can be used to compute the MAC operations in the subsequent clock cycles. This approach allows to significantly improve the processing rate of the PEs, since it greatly reduces the critical path of the circuit. This aspect is of utmost importance in the proposed unified transform architecture, because all the computations that are performed in the arithmetic module of the PEs are realized using integer arithmetic circuits with a relatively high resolution, as a result of the increased dynamic gains imposed by the higher order transform kernels considered in the state-of-the-art video standards (e.g. HEVC [4]).

Nevertheless, this procedure also imposes an explicit PE configuration stage prior to the computation of a new transform, whenever the architecture is reprogrammed to make use of a different transform kernel. In such cases, the PEs are also configured in a wavefront manner, just like in the dataflow model described in Section 4.1. The command that triggers such event is also sent through the top left corner PE, which then propagates it to the remaining PEs in both the horizontal and vertical directions. Since this operation requires only one single clock cycle and is performed in a pipelined fashion, it is possible to start the computation of a new transform operation in each PE on the clock cycle that immediately succeeds its reconfiguration stage. As a result, only when it is necessary to compute a new transform involving a kernel different from the one currently in use within the systolic array does the PE reprogrammable architectural feature increase the latency of the unified transform architecture in one single clock cycle.

The arithmetic module of the PEs makes use of an accumulator and of a specialized multiplication circuit to perform all the required transform operations. In this scope, the data values to be processed $\left(\mathrm{X}_{\mathrm{in}}\right)$ are placed at one of the inputs of the multiplier. Conversely, the partial value of the transform operation being computed ( $A C C$ in), which was calculated by an adjacent PE in the previous clock cycle, is placed at one of the inputs of the accumulator. Then, this partial value is updated with the result of the multiplication involving $X_{\text {in }}$ and the kernel value corresponding to the multiplier control word stored in the internal standing-data register of the PE, by also taking into consideration the sign information bit stored in the same internal standing-data register. The resulting value is stored in another internal standing-data register before being propagated to the following PEs in the array, in order to shorten the critical path of the architecture and to guarantee the systolic dataflow.

This highly flexible PE architecture allows the designer to easily adapt the PE functionality, so that multiple and distinct transform kernels can be supported. In fact, in order to design a new PE structure that is capable of supporting one or more transform kernels, it is only necessary to encode the considered kernel values in the ROM and develop a new multiplication circuit. By jointly exploiting this feature and the modularity and reconfigurable properties of the presented unified architecture, it is possible to realize several different transform cores addressing not only the subset of transforms that are used by a specific video standard but also more generic MST cores. In this work, all these properties were extensively exploited, in order to design a new set of PEs addressing the AVC, AVS, VC- 1 and HEVC standards. In addition, a multi-standard $\mathrm{PE}$ that is capable of processing the transforms defined in all these standards by using a resource-shared multiplication circuit was also developed. To better explain the procedures involved in the design of all these PEs, the following subsection presents a brief review of all the development stages of the proposed multi-standard PE, which is taken as a case study.

\subsection{Resource-shared multi-standard PE}

In order to fully support the computation of all the transforms defined in the AVC, AVS, VC-1 and HEVC standards, a multi-standard PE for the proposed unified transform architecture must be capable of computing all the MAC operations required by 28 different transforms (see Section 2). This involves 80 distinct transform kernel values in the range of -90 to +90 . However, only one of these constants is used by the PE to perform the required MAC operation at any moment. Consequently, this opens the opportunity to make use of time-multiplexed multiple constant multiplication (MCM) structures [35], in order to implement reduced-area and faster multipliers for the PE.

Although several different approaches can be considered to develop this class of multipliers based on the shift-and-add algorithm, the addition chain [36] method for multiplying by a constant and a directed acyclic graph (DAG) fusion algorithm similar to the one presented in [35] are adopted in this work. The first technique is used to reduce the number of additions, by allowing the results of the intermediate operations to be shifted and reused in arbitrary subsequent additions. For the considered type of MCM problems, where an input value must be multiplied by one of $p$ given preset constants, addition chains are also able to provide some potential 
extra savings, since they allow the sharing of common subexpressions for the computation of the $p$ constants. The considered DAG fusion algorithm is applied in a later development stage, in order to 'fuse' the addition chains of the several individual constants into a single network of adders, wired shifts and multiplexers, suitable for time-multiplexing. Furthermore, the application of this algorithm also guarantees that the resulting fused addition chain circuit only includes as many adders as the largest of its fundamental addition chains.

In what concerns the proposed multi-standard PE, which implements the architecture presented in Figure 2, the first step in the design of its time-multiplexed MCM consisted in the definition of a DAG, representing an optimal addition chain, for each one of the considered kernel values (i.e. the basis values of all the considered transform kernels presented in Table 1). The next design step aimed at finding and exploiting the similarities in all these graphs, in order to obtain the best composite DAG jointly representing the addition chains of all the individual DAGs. Such graph consists exclusively of additions, shifts and multiplexers, as it can be seen in Figure 4. Then, a proper hardware structure was devised to implement the DAG that was obtained in the previous design step. As it can be seen in Figure 5, such circuit requires only three adders and four $(2: 1)$ multiplexers, in order to perform all the MAC operations involved in the computation of the 28 transforms considered in the $\mathrm{AVC}, \mathrm{AVS}, \mathrm{VC}-1$ and HEVC

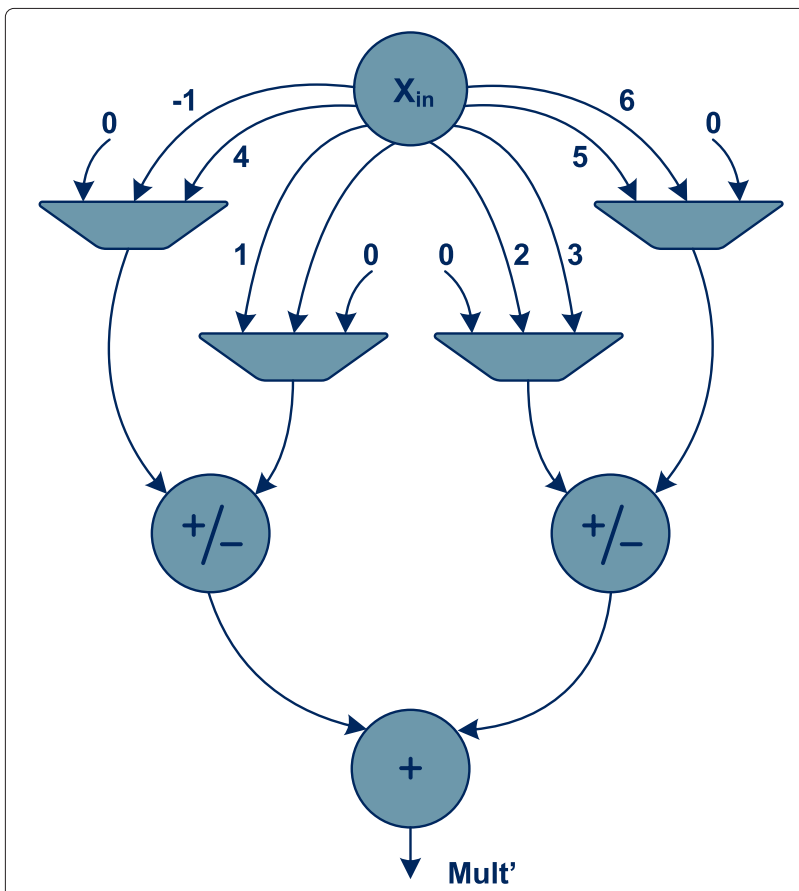

Figure 4 DAG of the MCM used in the arithmetic module of the multi-standard PE. standards. In the following design step, a 10-bit control word was defined for each one of the considered kernel values. These 10-bit words are used to command the operation of all the multiplexers and addition/subtraction circuits composing the hardware structure obtained in the previous design step. Such control words are presented (in hexadecimal notation) in Table 2 for the set of kernel values shown in Table 1. Finally, the contents of the ROM that is embedded in the control module of the proposed multi-standard PE were specified, by properly disposing all the multiplier control words in 11 different memory segments.

As it can be seen in Figure 6, each one of these memory segments concerns a distinct transform kernel of order$N$, which can be addressed by using the Type T signal, according to the data presented in Table 3. Within each memory segment, $N$ consecutive memory positions are occupied with the multiplier control words corresponding to the $N$ basis values of the considered transform kernel. This data is disposed in the same manner as its corresponding kernel values in the first column of the transform kernel matrix. As a result, in order to address and retrieve a given transform kernel value from the PE ROM, it is only necessary to combine the bits of the Type_T signal with those of the address value generated by the Multiplier Decoder block $\left(f^{\prime}\right)$, which represents the offset inside the memory segment.

Consequently, by using this memory layout to program the multi-standard PE ROM with all the multiplier control words required by the the AVC, AVS, VC- 1 and HEVC standards, it was possible to greatly reduce the complexity of the Multiplier Decoder in the PE.

\section{Experimental evaluation}

The advantages offered by the proposed unified transform architecture in the design of high-performance and dedicated MST cores were experimentally assessed using FPGA realizations. The implemented processing structures are based on a systolic array with $8 \times 8$ PEs, which was configured to support the computation of the transforms defined in the AVC, AVS and VC-1 standards, as well as the $4 \times 4$ and $8 \times 8$ HEVC transforms, by using the PEs presented in Section 4.2.

Naturally, alternative configurations of the proposed processing structure could have been similarly devised, in order to also enable the computation of the higher order transforms defined in the HEVC standard. In such cases, it would only be required to consider a different configuration for the presented architecture which, for efficiency reasons, should include a systolic array at least of the same dimension as the size of the greatest transform to be computed. Nonetheless, in the presented experimental evaluation, it was decided to consider implementations supporting only transforms of order- 4 and 


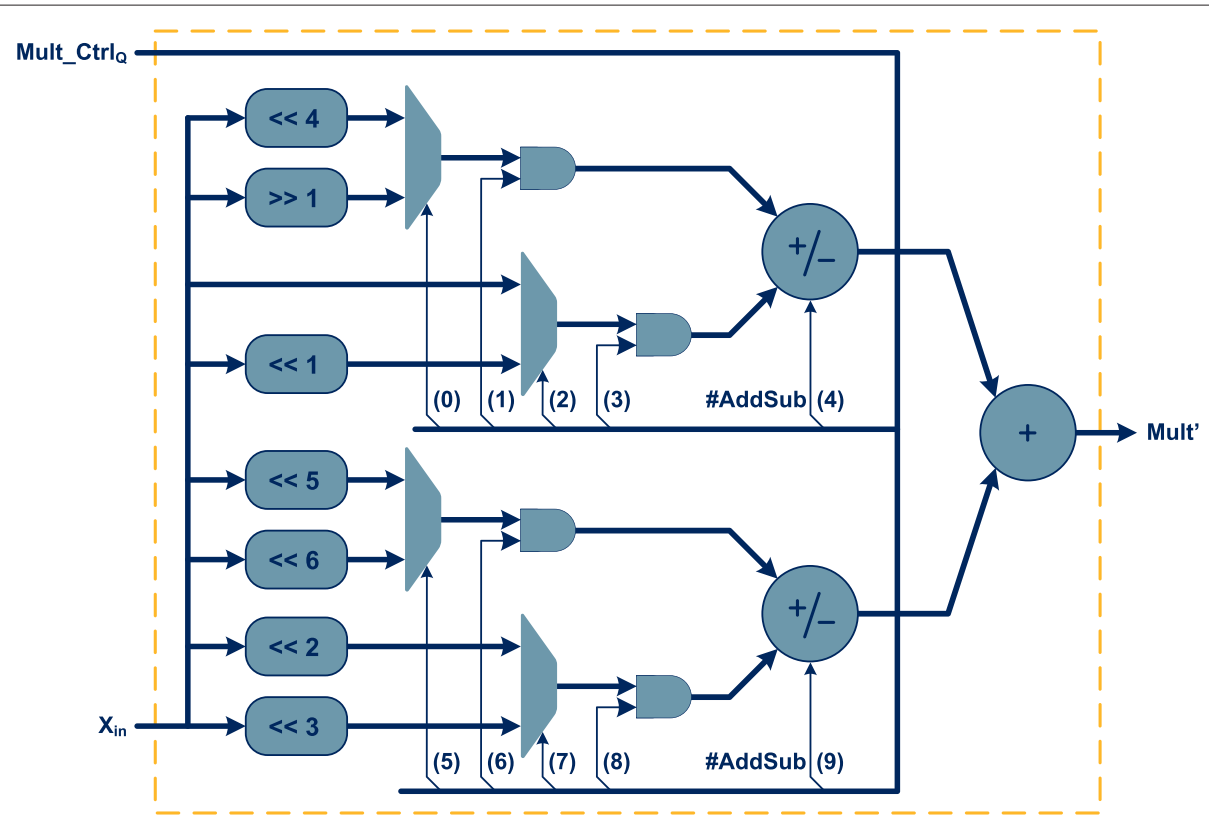

Figure 5 Architecture of the MCM used in the arithmetic module of the multi-standard PE.

order-8, because most of the existing alternative designs are based on this configuration setup.

It should be noted that the chosen architecture configuration does not compromise either the relevance of the proposed unified architecture or the benefits that it offers in terms of performance and functionality when compared with other alternative solutions, as is discussed in Section 5.2.

\subsection{FPGA implementation}

The considered hardware realizations were synthesized and implemented in a Xilinx Virtex-7 XC7VX485T FPGA device, by using the Xilinx ISE 13.1i tool chain and a parameterizable IEEE-VHDL description of the presented unified transform architecture and of all the proposed PEs. Such generic architectural descriptions follow a strict modular approach using independent and self-contained functional blocks, in order to comply with the scalability and multi-transform requirements of the architecture. Table 4 presents the implementation results of the devised PEs, while Table 5 summarizes the hardware realization of the considered MST core. All the presented results were obtained by performing a standard synthesis procedure, targeting performance optimized circuits.

The results presented in Table 4 demonstrate the reduced hardware cost of the devised PEs, since all the considered implementations require between 10 and 14 Virtex-7 slices. As it was expected, the multi-standard PE is the processing structure that requires more hardware resources, due to the increased flexibility that is offered to support the computation of all the transforms defined in the AVC, AVS, VC-1 and HEVC standards. Accordingly, it is also the processing structure presenting the lowest value for the maximum clock frequency, i.e. 285.3 $\mathrm{MHz}$. Again, this is a result of its ability to perform the transform computations involving 80 different kernel values, which requires not only a MCM with a longer latency, due to the usage of two adders interconnected in cascade (see Figure 5), but also arithmetic circuits with a larger bit width in the datapath of the PE (i.e. 38 bits for input values originally encoded with 16 bits), as a result of the increased dynamic gain of the HEVC transform kernels (i.e. 22 bits). Since the dynamic gains of the AVC, VC-1 and AVS transform kernels are relatively lower (i.e. 12, 13

Table 2 Multiplier control words for the AVC, AVS, VC-1 and HEVC transform kernels (see Table 1)

\begin{tabular}{lcccccccccc}
\hline Kernel value & $\frac{1}{2}$ & 1 & 2 & 3 & 4 & 6 & 8 & 12 \\
ROM word & $0 \times 3$ & $0 \times 8$ & $0 \times C$ & $0 \times 118$ & $0 \times 100$ & $0 \times 10 C$ & $0 \times 180$ & $0 \times 188$ & $0 \times 18 C$ & $0 \times 2 F D$ \\
\hline Kernel value & 15 & 16 & 18 & 36 & 50 & 64 & 75 & 83 \\
ROM word & $0 \times 1 A$ & $0 \times 2$ & $0 \times E$ & $0 \times 140$ & $0 \times 4 E$ & $0 \times 60$ & $0 \times 285$ & $0 \times 17 A$ & $0 \times 1 E A$ \\
\hline
\end{tabular}




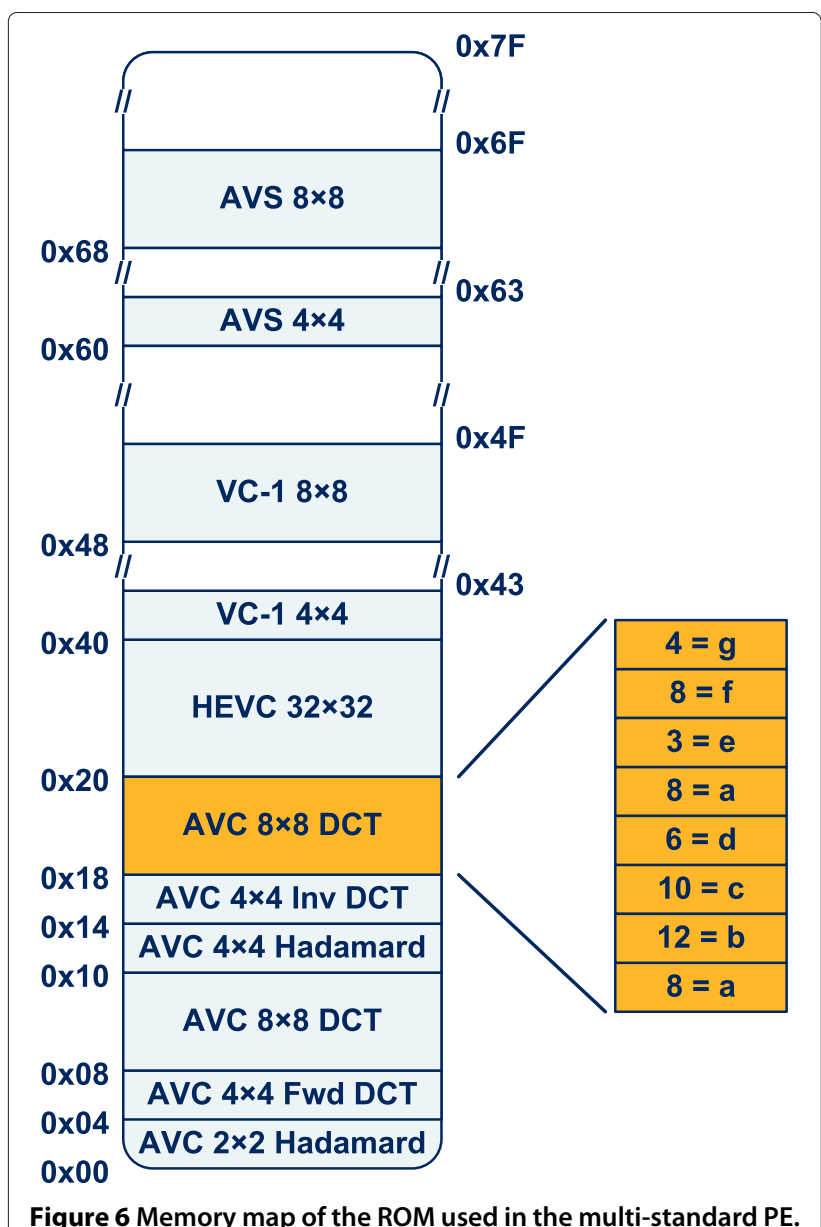

Figure 6 Memory map of the ROM used in the multi-standard PE.

and 14 bits, respectively), the bit width of the arithmetic circuits composing the datapaths of the PEs that were designed to exclusively address such standards is therefore smaller.

As it can be seen in Table 4, such characteristic allows these PEs to be operated using relatively higher clock frequencies. The highest clock frequency corresponds to the AVS PE, due to the simplicity and the lower latency of the involved MCM that includes only a single adder/subtractor. Nevertheless, all the clock frequency values that are presented in Table 4 clearly evidence the high processing rates that are offered by all the devised PEs. In fact, from these results, it can concluded that any of these PEs can be used in the processing of video sequences with resolutions as high as $7,680 \times 4,320$ pixels (i.e. the $8 \mathrm{k}$ Ultra inition Television (UHDTV) format) in real time.

This conclusion can be easily extrapolated from Table 5 , which shows that the implemented MST core is able not only to compute up to 17.9 giga operations per second (GOPS) but also to offer a sustained processing throughput of about $2.2 \times 10^{9}$ samples per second (GSamp/s),
Table 3 Encoding of the Type_T signal for the implementation of the MST core

\begin{tabular}{ll}
\hline Type_T & Transform kernel \\
\hline 0 & AVC $2 \times 2$ Hadamard \\
1 & AVC $4 \times 4$ Hadamard \\
2 & AVC $4 \times 4$ forward DCT \\
3 & AVC $8 \times 8$ forward DCT \\
4 & AVC $2 \times 2$ Hadamard \\
5 & AVC $4 \times 4$ Hadamard \\
6 & AVC $4 \times 4$ inverse DCT \\
7 & AVC $8 \times 8$ inverse DCT \\
8 & HEVC $4 \times 4$ DCT \\
9 & HEVC $8 \times 8$ DCT \\
10 & VC-1 $4 \times 4$ DCT \\
11 & VC-1 $4 \times 8$ DCT \\
12 & VC-1 $8 \times 4$ DCT \\
13 & VC-1 $8 \times 8$ DCT \\
14 & AVS $4 \times 4$ DCT \\
15 & AVS $8 \times 8$ DCT
\end{tabular}

by using a clock frequency of $279.6 \mathrm{MHz}$. These relatively high performance levels are very similar to the ones provided by the design that was proposed in [7], despite such processing structure is only capable of computing the transforms defined in the AVC and HEVC standards. This result was already expected and is due to the following two reasons: (i) the pipelined processing nature of the presented unified transform architecture and (ii) the quite similar latency values imposed by the devised PEs, which is a result of their highly optimized and almost identical internal hardware structure.

The results that are presented in Table 5 also demonstrate the quite reduced hardware cost of the devised proof-of-concept MST architecture, while offering a remarkable processing rate. As it can be seen, less than $4 \%$ of the total hardware resources available in the adopted medium-size FPGA device were used in the realization of

Table 4 Implementation results of the devised PEs in a Xilinx Virtex-7 FPGA

\begin{tabular}{lccc}
\hline Processing structure & Registers & LUTs & $\begin{array}{c}\text { Maximum frequency } \\
{[\mathbf{M H z}]}\end{array}$ \\
\hline PE for AVC & 67 & 124 & 371.5 \\
PE for AVS & 66 & 111 & 395.3 \\
PE for VC-1 & 65 & 160 & 302.0 \\
PE for HEVC & 79 & 253 & 289.3 \\
Multi-standard PE & 80 & 299 & 285.3 \\
\hline
\end{tabular}


Table 5 Implementation results of the proof-of-concept MST core in a Xilinx Virtex-7 FPGA

\begin{tabular}{lccc}
\hline Processing structure & Registers & LUTs & $\begin{array}{c}\text { Maximum frequency } \\
\text { [MHz] }\end{array}$ \\
\hline$\boxminus$ MST core & 7,309 & 21,568 & 279.4 \\
$\boxplus$ Input buffer & 1,681 & 1,265 & 388.7 \\
$\boxminus$ Array with 8 8 PEs & 5,332 & 18,624 & 284.1 \\
$\boxplus P E$ & 80 & 299 & 285.3 \\
$\boxplus$ Transposition switch & 1,362 & 2,161 & 417.4 \\
$\boxplus$ Control unit & 97 & 77 & 573.1 \\
\hline
\end{tabular}

the considered transform core. Almost all of these hardware resources (about $86 \%$ ) are used to implement the 2-D systolic array, with each of its $8 \times 8$ multi-standard PEs requiring at most 14 slices. Therefore, it can be observed that while the hardware cost of the presented unified architecture mostly results from the adopted configuration for its systolic array, its maximum clock frequency is only limited by the critical path of the PEs. Consequently, it can be expected that very similar performance levels will be attained for other configurations of the proposed unified architecture making use of larger systolic arrays (e.g. an array with $32 \times 32 \mathrm{PEs}$, to allow the computation of the higher order transforms defined in HEVC).

\subsection{Comparative analysis}

In order to further demonstrate the advantages that are offered by the presented unified architecture in the design of video codecs supporting the most recent and stateof-the-art video standards, several different alternative designs recently described in the literature for this domain were reviewed. Due to the diverse set of technologies that are considered in the implementation of these designs, as well as to the different functionalities that are presented by each implementation and the distinct considerations that might have been adopted, this analysis mostly focused on a comparison of the functionalities and computational rates offered by the various structures. Table 6 summarizes the results of such comparative analysis for a subset of the most related and prominent designs that were evaluated. Note that the presented data concerns only to the implementation of the transform computation module(s) of the considered transform cores.

A straightforward analysis of the data presented in Table 6 clearly shows that only a few of the reviewed designs are able to offer a multi-standard functionality. In addition, it also evidences that only the designs presented in $[7,27]$ are able to support the computation of the HEVC transforms. Nevertheless, it should be noted that while the architecture presented in [7] is capable of realizing the complete set of transforms defined in the standard, the one proposed in [27] is able to compute only the HEVC inverse transforms. Therefore, the application domain of this processing structure is limited to the design of high-performance decoders, being unsuitable to implement video encoders. This characteristic is common to many of the designs enumerated in Table 6, since they are able to compute only a subset of the transforms defined in one or multiple video standards [23,24,26,28,29,33]. In fact, to the best of the authors' knowledge, the MST architecture herein proposed is one of the first structures that is able to compute the complete set of transforms adopted in the AVC, AVS, VC-1 and HEVC standards.

Table 6 Functional and performance comparison with other architectures

\begin{tabular}{|c|c|c|c|c|c|c|c|c|c|}
\hline \multirow{2}{*}{ Design } & \multirow{2}{*}{ Technology } & \multirow{2}{*}{$\begin{array}{l}\text { Maximum frequency } \\
{[\mathrm{MHz}]}\end{array}$} & \multirow{2}{*}{$\begin{array}{l}\text { Latency } \\
\text { [ns] }\end{array}$} & \multirow{2}{*}{$\begin{array}{l}\text { Throughput } \\
\text { [GSamp/s] }\end{array}$} & \multicolumn{4}{|c|}{ Supported standards } & \multirow{2}{*}{ Applications } \\
\hline & & & & & AVC & VC-1 & AVS & HEVC HEVC & \\
\hline [33] & $180 \mathrm{~nm}$ & 146.0 & 438.4 & 0.2 & $\sqrt{ }$ & $\sqrt{ }$ & $\sqrt{ }$ & & HD 1080p \\
\hline [15] & $180 \mathrm{~nm}$ & 100.0 & 20.0 & 0.8 & $\sqrt{ }$ & & & & Digital Cinema 2k \\
\hline [24] & $180 \mathrm{~nm}$ & 230.9 & 34.7 & 1.9 & $\sqrt{ }$ & & & & 8k UHDTV (4320p) \\
\hline [23] & $180 \mathrm{~nm}$ & 300.0 & 53.3 & 2.4 & $\sqrt{ }$ & & & & HD 720p \\
\hline [25] & $180 \mathrm{~nm}$ & 200.0 & 20.0 & 4.1 & $\sqrt{ }$ & & & & HD 2160p \\
\hline [28] & $180 \mathrm{~nm}$ & 211.4 & 56.8 & 0.2 & $\sqrt{ }$ & $\sqrt{ }$ & $\sqrt{ }$ & & HD 1080p \\
\hline [29] & $130 \mathrm{~nm}$ & 384.0 & 83.3 & 0.8 & $\sqrt{ }$ & $\sqrt{ }$ & $\sqrt{ }$ & & 4k UHDTV (2160p) \\
\hline$[31]$ & $130 \mathrm{~nm}$ & 100.0 & $1,000.0$ & 0.4 & $\sqrt{ }$ & $\sqrt{ }$ & $\sqrt{ }$ & & HD 1080p \\
\hline [26] & $130 \mathrm{~nm}$ & 300.0 & 70.0 & 3.7 & $\sqrt{ }$ & & & & 4k UHDTV (2160p) \\
\hline$[27]$ & $130 \mathrm{~nm}$ & 350.0 & - & 1.4 & $\sqrt{ }$ & $\sqrt{ }$ & $\sqrt{ }$ & $\sqrt{ }$ & 4k UHDTV (2160p) \\
\hline [34] & Virtex-4 & 110.8 & 144.4 & 0.9 & $\sqrt{ }$ & $\sqrt{ }$ & $\sqrt{ }$ & & 4k UHDTV (2160p) \\
\hline [24] & Virtex-4 & 133.5 & 59.9 & 1.1 & $\sqrt{ }$ & & & & 4k UHDTV (2160p) \\
\hline [7] & Virtex-7 & 280.6 & 57.0 & 2.2 & $\sqrt{ }$ & & & $\sqrt{ }$ & 8k UHDTV (4320p) \\
\hline Proposed & Virtex-7 & 279.4 & 57.2 & 2.2 & $\sqrt{ }$ & $\sqrt{ }$ & $\sqrt{ }$ & $\sqrt{ }$ & $8 k$ UHDTV (4320p) \\
\hline
\end{tabular}


In what concerns the offered performance levels, the comparison that is presented in Table 6 clearly shows that the implemented transform core offers one of the highest computation rates (2.2 GSamp/s), despite being one of the few designs with enhanced multi-standard transform computation capabilities. In fact, it can be observed that this design outperforms almost all the other considered architectures by about 1.8 times. The only exceptions are the designs presented in $[7,23,26]$. However, these faster processing structures are able to compute only a quite reduced subset of the transforms supported by the devised proof-of-concept transform core, since they were either specifically designed to accelerate the inverse $8 \times 8$ DCT [23], optimized for the implementation of the forward transform coding path of a video encoder [26], or only support the AVC and HEVC standards [7].

The results presented in Table 6 also demonstrate that the proposed unified transform architecture allows to design MST cores that impose very small latency in the processing of the video bit streams, when compared to the majority of the considered alternative designs. This is a quite important aspect when real-time operation is considered, since the latency that is imposed to the processing of each video frame may compromise the operation of the codec in 'live' mode.

\section{Conclusions}

A high-performance unified architecture for the computation of a vast set of 2-D transforms adopted by several modern video standards is proposed in this paper. To the best of the authors' knowledge, this is one of the first structures that is capable of computing all the DCTs of different orders (e.g. order-4, order-8, order-16 and order-32) that are adopted in the AVC, AVS, VC-1 and HEVC standards. Contrasting to other dedicated transform cores, the presented architecture is supported on a completely configurable and scalable structure, capable of providing the processing rates that are required to compute, in real time, all the transforms in HD video sequences. When prototyped in a Xilinx Virtex-7 FPGA device, an MST core based on the proposed unified architecture is able to compute up to 17.9 GOPS and offer a sustained throughput of about $2.2 \times 10^{9}$ samples per second, by using a clock frequency of $279.6 \mathrm{MHz}$. Such relatively high performance levels allow this hardware structure, supporting the AVC, AVS, VC-1 and HEVC standards, to process in real-time $8 \mathrm{k} \operatorname{UHDTV}(7,680 \times 4,320)$ sequences at a frame rate of $30 \mathrm{fps}$.

\section{Competing interests}

The authors declare that they have no competing interests.

\section{Acknowledgements}

This work was supported by national funds through Fundação para a Ciência e a Tecnologia (FCT) under the project PEst-OE/EEI/LA0021/2013 and by the Programa de apoio à Formação avançada de docentes do Ensino Superior
Politécnico (PROTEC) programme funds, under the research grant SFRH/PROTEC/50152/2009.

\section{Author details}

${ }^{1}$ INESC-ID, Rua Alves Redol, 9, Lisbon 1000-029, Portugal. ${ }^{2}$ IST, Universidade de Lisboa, Avenida Rovisco Pais, 1, Lisbon 1049-001, Portugal. ${ }^{3}$ ISEL, Instituto Politécnico de Lisboa, Rua Conselheiro Emídio Navarro, 1, Lisbon 1959-007, Portugal.

Received: 1 March 2014 Accepted: 30 June 2014

Published: 11 July 2014

\section{References}

1. TWiegand, G Sullivan, G Bjntegaard, A Luthra, Overview of the H.264/AVC video coding standard. IEEE Trans. Circ. Syst. Video Tech. 13(7), 560-576 (2003)

2. L Yu, S Chen, J Wang, Overview of AVS-video coding standards. Signal Process. Image Comm. 24(4), 247-262 (2009)

3. S Srinivasan, SL Regunathan. Vis. Commun. Image Process. $\mathbf{5 9 6 0}$ 720-728 (2005)

4. K Ugur, K Andersson, A Fuldseth, G Bjontegaard, LP Endresen, J Lainema, A Hallapuro, J Ridge, D Rusanovskyy, C Zhang, A Norkin, C Priddle, T Rusert, J Samuelsson, R Sjoberg, Z Wu, High performance, low complexity video coding and the emerging HEVC standard. IEEE Trans. Circ. Syst. Video Tech. 20(12), 1688-1697 (2010)

5. Z-N Li, MS Drew, Fundamentals of Multimedia, 1st. (Prentice Hall, New Jersey, 2003)

6. KR Rao, P Yip, Discrete Cosine Transform: Algorithms, Advantages, Applications. (Academic Press, San Diego, 1990)

7. T Dias, N Roma, L Sousa, High performance multi-standard architecture for DCT computation in H.264/AVC high profile and HEVC codecs, in Conference on Design \& Architectures for Signal and Image Processing (Cagliari, 2013), pp. 14-21

8. W-K Cham, Development of integer cosine transforms by the principle of dyadic symmetry. Commun. Speech Vision IEEE Proc. I. 136(4), 276-282 (1989)

9. C Zhang, L Yu, J Lou, W-K Cham, J Dong, The technique of prescaled integer transform: concept, design and applications. IEEE Trans. Circ. Syst. Video Tech. 18(1), 84-97 (2008)

10. J-J Lee, S Park, N Eum, Design of application specific processor for H.264 inverse transform and quantization, in International SoC Design Conference, vol. 2 (Busan, 2008), pp. 57-60

11. NT Ngo, TTT Do, TM Le, YS Kadam, A Bermak, ASIP-controlled inverse integer transform for H.264/AVC compression, in The 19th IEEE/IFIP International Symposium on Rapid System Prototyping (Monterey, 2008), pp. 158-164

12. J Shan, C Chen, E Yang, High performance 2-D IDCT for image/video decoding based on FPGA, in International Conference on Audio, Language and Image Processing (Shanghai, 2012), pp. 33-38

13. W-H Chen, C Smith, S Fralick, A fast computational algorithm for the discrete cosine transform. IEEE Trans. Comm. 25(9), 1004-1009 (1977)

14. C-P Fan, Fast 2-dimensional $4 \times 4$ forward integer transform implementation for H.264/AVC. IEEE Trans. Circuits Syst. II. 53(3), 174-177 (2006)

15. K-H Chen, J-I Guo, J-S Wang, A high-performance direct 2-D transform coding IP design for MPEG-4AVC/H.264. IEEE Trans. Circ. Syst. Video Tech. 16(4), 472-483 (2006)

16. S Shen, W Shen, Y Fan, X Zeng, A unified forward/inverse transform architecture for multi-standard video codec design. IEICE Trans. Fund. Electron. Comm Comput. Sci. 96-A(7), 1534-1542 (2013)

17. JS Park, WJ Nam, SM Han, S Lee, 2-D large inverse transform (16×16, $32 \times 32$ ) for HEVC (high efficiency video coding). J. Semicond Technol. Sci. 12(2), 203-211 (2012)

18. L Ling-Zhi, Q Lin, P Meng-tian, J Li, A 2-D forward/inverse integer transform processor of $\mathrm{H} .264$ based on highly-parallel architecture, in 4th IEEE International Workshop on System-on-Chip for Real-Time Applications (Banff, 2004), pp. 158-161

19. C-P Fan, Cost-effective hardware sharing architectures of fast $8 \times 8$ and $4 \times 4$ integer transforms for H.264/AVC, in IEEE Asia Pacific Conference on Circuits and Systems (Singapore, 2006), pp. 776-779 
20. RB Are, K Rajan, An RNS based transform architecture for H.264/AVC, in 2008 IEEE Region 10 Conference TENCON (Hyderabad, 2008), pp. 1-6

21. C-C Sun, P Donner, J Götze, VLSI implementation of a configurable IP core for quantized discrete cosine and integer transforms. Int. J. Circ. Theor. Appl. 40(11), 1107-1126 (2012)

22. Y-H Chen, J-N Chen, T-Y Chang, C-W Lu, High-throughput multistandard transform core supporting MPEG/H.264/NC-1 using common sharing distributed arithmetic. IEEE Trans. Very Large Scale Integrat. Syst. PP(99), 1-1 (2013)

23. C Jiang, N Yu, M Gu, A novel VLSI architecture of $8 \times 8$ integer DCT based on H.264/AVC FRext, in 3rd International Symposium on Knowledge Acquisition and Modeling (Wuhan, 2010), pp. 59-62

24. TTT Do, TM Le, High throughput area-efficient SoC-based forward/inverse integer transforms for H.264/AVC, in IEEE International Symposium on Circuits and Systems (Paris, 2010), pp. 4113-4116

25. W Hwangbo, C-M Kyung, A multitransform architecture for H.264/AVC High-Profile coders. IEEE Trans. Multimed. 12(3), 157-167 (2010)

26. M Gu, N Yu, C Jiang, W Lu, Hardware prototyping for various transforms in H.264 high profile. J. Inform. Comput. Sci. 8(1), 119-128 (2011)

27. S Shen, W Shen, Y Fan, X Zeng, A unified 4/8/16/32-point integer IDCT architecture for multiple video coding standards, in IEEE International Conference on Multimedia and Expo (Melbourne, 2012), pp. 788-793

28. M Martuza, C McCrosky, K Wahid, A fast hybrid DCT architecture supporting H.264, VC-1, MPEG-2, AVS and JPEG codecs, in 11th International Conference on Information Science, Signal Processing and Their Applications (Montreal, 2012), pp. 545-549

29. THo, T Le, K Vu, S Mochizuki, K Iwata, K Matsumoto, H Ueda, A 768 megapixels/sec inverse transform with hybrid architecture for multi-standard decoder, in IEEE International Conference on ASIC (Xiamen, 2011), pp. 71-74

30. Y-K Lai, Y-F Lai, A reconfigurable IDCT architecture for universal video decoders. IEEE Trans. Consum. Electron. 56(3), 1872-1879 (2010)

31. K Wang, J Chen, W Cao, Y Wang, L Wang, J Tong, A reconfigurable multi-transform VLSI architecture supporting video codec design. IEEE Trans. Circ. Syst. II. 58(7), 432-436 (2011)

32. $\mathrm{YLi}, \mathrm{Y} \mathrm{He}, \mathrm{S}$ Mei, $\mathrm{A}$ highly parallel joint VLSI architecture for transforms in H.264/AVC. J. Signal Process. Syst. 50(1), 19-32 (2008)

33. KWahid, M Martuza, M Das, C Mc Crosky, Resource shared architecture of multiple transforms for multiple video codecs, in 24th Canadian Conference on Electrical and Computer Engineering (Niagara Falls, 2011), pp. 947-950

34. G Liu, An area-efficient IDCT architecture for multiple video standards, in 2nd International Conference on Information Science and Engineering (Hangzhou, 2010), pp. 3518-3522

35. P Tummeltshammer, JC Hoe, M Puschel, Time-multiplexed multiple-constant multiplication. IEEE Trans. Comput. Aided Des. Integrated Circ. Syst. 26(9), 1551-1563 (2007)

36. F Bergeron, J Berstel, S Brlek, Efficient computation of addition chains. J. de théorie des nombres de Bordeaux. 6(1), 21-38 (1994)

\section{Submit your manuscript to a SpringerOpen ${ }^{\circ}$ journal and benefit from:}

- Convenient online submission

- Rigorous peer review

- Immediate publication on acceptance

- Open access: articles freely available online

- High visibility within the field

- Retaining the copyright to your article 